\title{
Microstructural deformation behaviour in polystyrene-based compatible polymer blend systems
}

\author{
Byoung Chul Chun* and Ronald Gibala \\ The Macromolecular Research Center, Department of Materials Science and Engineering, \\ The University of Michigan, Ann Arbor, MI 48109, USA \\ (Received 28 July 1993)
}

\begin{abstract}
Microstructural deformation behaviour in polystyrene-based compatible polymer blend systems was studied using transmission electron microscopy (TEM) and microdensitometry. Four different binary compatible blend systems were employed and characterized in this investigation: polystyrene (PS) and poly(2,6-dimethyl1,4-phenylene oxide)(PPO), PS and poly(vinyl methyl ether)(PVME), PS and poly( $\alpha$-methylstyrene)(P $\alpha \mathrm{MS})$, and PPO and P $\alpha$ MS. Individual craze and shear deformation zone (DZ) microstructures were examined by TEM. For TEM observations, specimens deformed in situ on a TEM grid (loaded condition) were utilized. TEM micrographs showed that, for PS/PPO blends, deformation mode transition from crazing to shear DZ occurred around 25\% PPO inclusion. For PS/PVME blends, this transition occurred around $20 \%$ PVME inclusion. For PS/P $\propto$ MS blends, the deformation mode was totally controlled by crazing regardless of composition. For PPO/P $\alpha$ MS blends, deformation mode transition from shear $\mathrm{DZ}$ to crazing occurred around 25\% P $\alpha \mathrm{MS}$ inclusion. Quantitative analyses of these crazes and shear DZs were conducted utilizing microdensitometry of the TEM negatives in the manner developed by Lauterwasser and Kramer. From the microdensitometry, molecular parameters such as fibril extension ratios $(\lambda s)$ were determined. Microdensitometry results showed that $\lambda$ decreased as the PPO content increased in the PS/PPO blends, and, for $100 \%$ PPO, only shear DZs were observed. For PS/PVME blends, $\lambda$ also decreased as the PVME content increased. For PS/P $\alpha \mathrm{MS}$ and PPO/P $\alpha \mathrm{MS}$ blends, $\lambda$ increased as the P $\alpha \mathrm{MS}$ content increased. These results were analysed in terms of existing entanglement and intermolecular interaction models in compatible blends. From this analysis, it is concluded that the overall microstructural deformation behaviour of binary compatible blends cannot be fully explained by either entanglement density or intermolecular interaction model alone. Rather, the combined entanglement density and intermolecular interaction model can explain the microstructural deformation behaviour in binary compatible blends well.
\end{abstract}

(Keywords: compatible blend; polystyrene; microstructural deformation)

\section{INTRODUCTION}

Ever since the introduction of Noryl ${ }^{\circledR}$, a commercial thermoplastic resin, by General Electric Co. in the mid-1960s ${ }^{1}$, there has been a growing interest in polymer blends in both industry and academia. Polymer blends represent a new source of materials available from the existing homopolymers. The existing properties of a homopolymer can be altered by introducing a second homopolymer. Even though there is a wealth of information available on polymer blends, the operating molecular mechanism that controls the deformation behaviour in compatible blends is not very well understood. Two types of plastic deformation modes are available in high-molecular-weight glassy amorphous polymer blends: one is crazing, and the other is shear banding.

Deformation mode changes, i.e. crazing to shear banding transition phenomena, can be examined using compatible blends of brittle and ductile homopolymers.

* To whom correspondence should be addressed. Present address: Department of Polymer Engineering, The University of Suwon, San 2-2, Wawu-ri, Bongdam-myon, Hwasung-gun, Kyonggi-do, Korea
At present, there are several different models available describing the mechanism of competition between crazing and shear yielding ${ }^{2-6}$. Among these models, two are generally regarded as important. One is based on the specific intermolecular interactions between the constituent polymers ${ }^{2,3}$, and the other is based on the entanglement density of the constituent polymers ${ }^{6}$.

The objective of this investigation is to obtain additional results with which to test the molecular models used to explain the deformation behaviour in compatible blends. In order to accomplish this objective, four different homopolymers were selected: polystyrene (PS), poly(2,6-dimethyl-1,4-phenylene oxide) (PPO), poly(vinyl methyl ether), (PVME) and poly ( $\alpha$-methylstyrene) (P $\alpha$ MS). Binary compatible polymer blends of PS/PPO, PS/PVME, $\mathrm{PS} / \mathrm{P} \alpha \mathrm{MS}$ and $\mathrm{PPO} / \mathrm{P} \alpha \mathrm{MS}$ were prepared and characterized. The reasons for choosing the above four blend systems are as follows: (i) All four blends are known to be compatible blends ${ }^{7-10}$. (ii) PS and P $\alpha$ MS have brittle properties, whereas PPO and PVME have ductile properties, thereby providing us with more information on the ductile-brittle transition phenomenon than in the previous investigations $\mathrm{s}^{2-6}$. (iii) The molecular weights of the PS, PPO and PVME samples chosen for this 
investigation are such that they are much higher than the critical molecular weight $\left(M_{\mathrm{c}}=\right.$ critical molecular weight for forming a stable entanglement network). In contrast, the P $\alpha$ MS sample has a molecular weight similar to its $M_{c}$; this allows us to get information about the $M_{c}$ effect on the deformation behaviour in compatible blends.

\section{EXPERIMENTAL}

\section{Materials}

Information concerning the four homopolymers used to prepare the compatible polymer blends in this study is presented in Table 1. PS was obtained through the courtesy of Dow Chemical Corporation as Dow 685. PPO was obtained through the courtesy of General Electric Corporation. P $\alpha \mathrm{MS}$ and PVME were purchased from Aldrich Chemical Co. and Polyscience Inc., respectively.

\section{Sample preparation}

A laboratory-scale two-roll mill was employed for blend preparations. The front roll was maintained at $50^{\circ} \mathrm{C}$ above the glass transition temperatures of the homopolymers used, while the rear roll was maintained at approximately $30^{\circ} \mathrm{C}$ below the temperature of the front roll. Initially, the homopolymers were placed on the front roll for $3 \mathrm{~min}$ to facilitate processing. Then the roll mill was started and another homopolymer was added. The milling was continued for $5 \mathrm{~min}$. Occasionally the molten blend was scraped out from the roll, wrapped several times and milled back to the roll. After 5 min of milling, the molten blend was scraped out and cut into specimens approximately $2 \mathrm{~cm} \times 2 \mathrm{~cm}$ in size. These pieces were then put into a grinding mill, and a small pellet-sized sample was obtained. These pellets were compression moulded at $50^{\circ} \mathrm{C}$ above their glass transition temperatures and air-cooled to room temperature. For PS/PVME blends, more than 35\% PVME inclusion caused the sample to be too ductile for tensile testing, whereas, for $\mathrm{PS} / \mathrm{P} \alpha \mathrm{MS}$ and $\mathrm{PPO} / \mathrm{P} \alpha \mathrm{MS}$ blends, more than $50 \%$ $\mathrm{P} \propto \mathrm{MS}$ inclusion caused the sample to be too brittle for tensile testing to initiate crazing or shear banding. Thus, all blend samples were manufactured within these compositions. Compositions of all blend samples used in this investigation are listed in Table 2.

\section{TEM sample preparation}

For the transmission electron microscope (TEM) observation, two sets of samples were used. The first set of samples were prepared from tensile fractured bulk samples. These samples, which contain crazes or shear bands, were microtomed to a thickness of $200 \mathrm{~nm}$ (using a Reichert-Jung ultramicrotome), placed on a 300 mesh TEM copper grid, and observed directly in the TEM (unloaded condition). The second set of samples were prepared from undeformed bulk samples. These samples were carefully microtomed, and a thin section (thickness $200 \mathrm{~nm}$ ) was placed on a 300 mesh TEM copper grid. This copper grid was stretched using a strain rig to initiate crazes or shear deformation zones (DZs) and observed directly in the TEM (loaded condition). The Zeiss transmission electron microscope (EM-10A) was used with an operating voltage of $100 \mathrm{kV}$.

\section{Microdensitometry}

For the quantitative analyses of individual crazes or shear DZs, microdensitometer scanning of the TEM negatives was performed using a Joyce-Loebl

Table 2 Compositions of the blend samples used in this investigation (weight percent)

\begin{tabular}{lcccccc}
\hline 1. PS/PPO & $100 / 0$ & $75 / 25$ & $50 / 50$ & $25 / 75$ & $0 / 100$ & \\
2. PS/PVME & $95 / 5$ & $90 / 10$ & $85 / 15$ & $80 / 20$ & $75 / 25$ & $65 / 35$ \\
3. PS/P $\alpha M S$ & $75 / 25$ & $50 / 50$ & & & & \\
4. PPO/P $\alpha M S$ & $75 / 25$ & $50 / 50$ & & & &
\end{tabular}

Table 1 Basic properties of the four homopolymers used in preparing the blends for this investigation

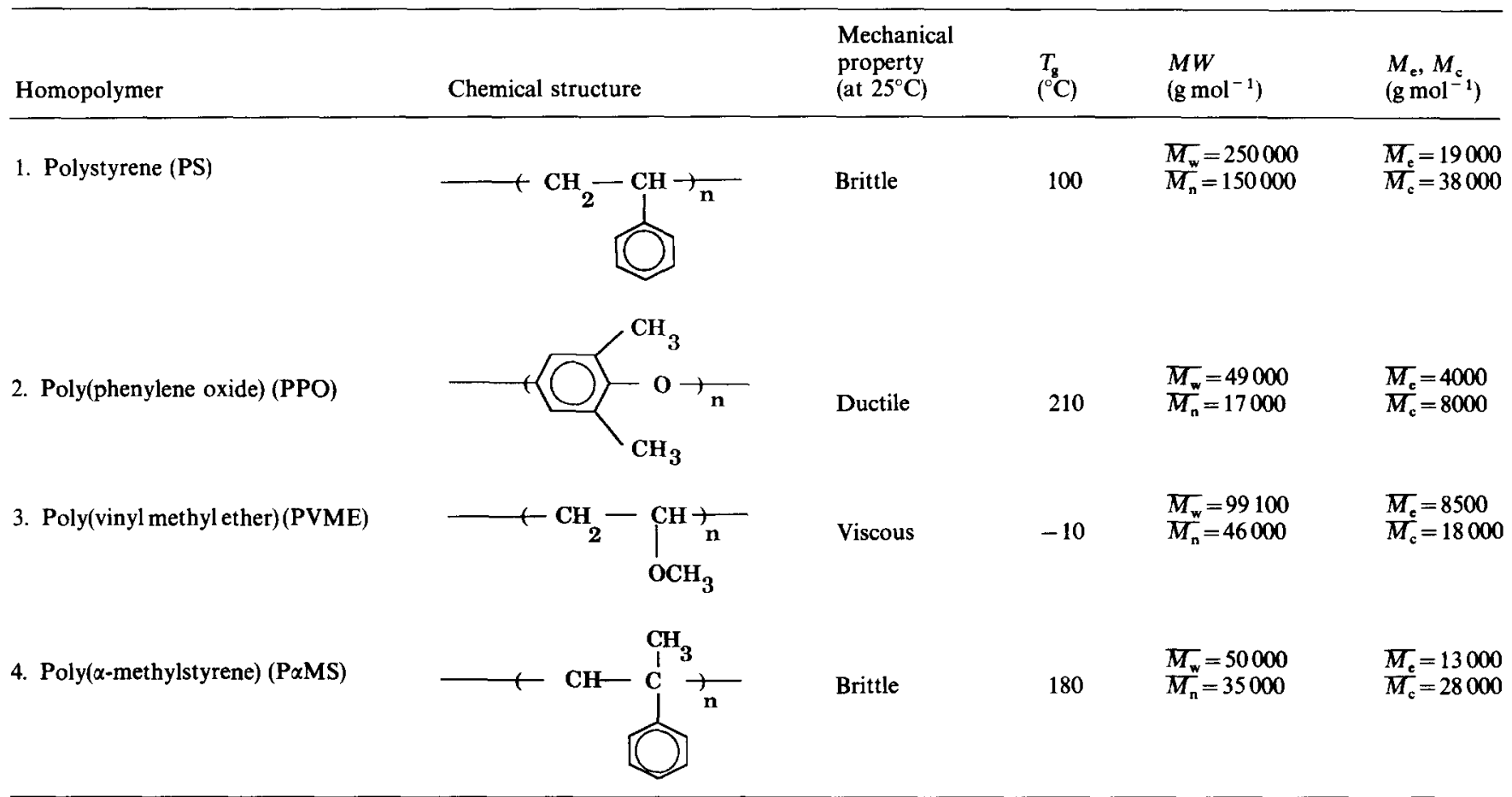


microdensitometer. From the calibration samples, relative optical densities of unity and zero were determined. With respect to these baseline densities, measurements of the optical densities of the crazes and shear DZs were carried out. The detailed experimental procedure is explained in Lauterwasser and Kramer's paper $^{11}$. An equation developed by the above authors was used to calculate fibril extension ratios of crazes and shear DZs. At least five TEM negatives of each composition were used to measure the fibril extension ratios, and the average values were used for plotting the data.

\section{RESULTS AND DISCUSSION}

\section{PS/PPO blends}

Figure 1 shows TEM micrographs of PS/PPO blends. Figures $l a$ and $l b$ show the craze microstructure of the unloaded $100 \%$ PS microtomed from the tensile fractured bulk sample, and the loaded $100 \%$ PS microtomed from undeformed bulk sample and later stretched on a strain rig, respectively. Figure la shows an irregular or even buckled structure of fibrils and voids, whereas Figure $1 b$ shows a regular alternating structure of extended fibrils and voids. In Figure la, there is also evidence of craze fibril tearing caused by the glass knife (sometimes referred to as a glass-knife mark) produced during the ultramicrotoming procedure. This glass-knife mark is shown as horizontal lines in Figure la. Figure $1 b$ also shows crack propagation through the craze, and the crack oscillates between craze/bulk boundaries and results in well known patch patterns on the fracture surface. According to Brown and $\mathrm{Kramer}^{12}$, in order to measure $\lambda$ or its inverse value, the fibril volume fraction $\left(v_{\mathrm{f}}\right)$, it is necessary to obtain a craze microstructure in the loaded condition. In the case of unloaded fibrils, $\lambda$ has a lower value than the actual fibril extension ratio owing to fibril retraction. Alternatively, Kambour ${ }^{13}$ also measured the void content of a PS craze and obtained a value of around $40 \%$ by making measurements in the unloaded state. This unloaded state probably resulted in a smaller void content than Kramer and coworkers' results $^{6,14,15}$. All other blend samples showed a similar behaviour for both unloaded (tearing and buckling of fibrils) and loaded samples (regular pattern of fibrils and

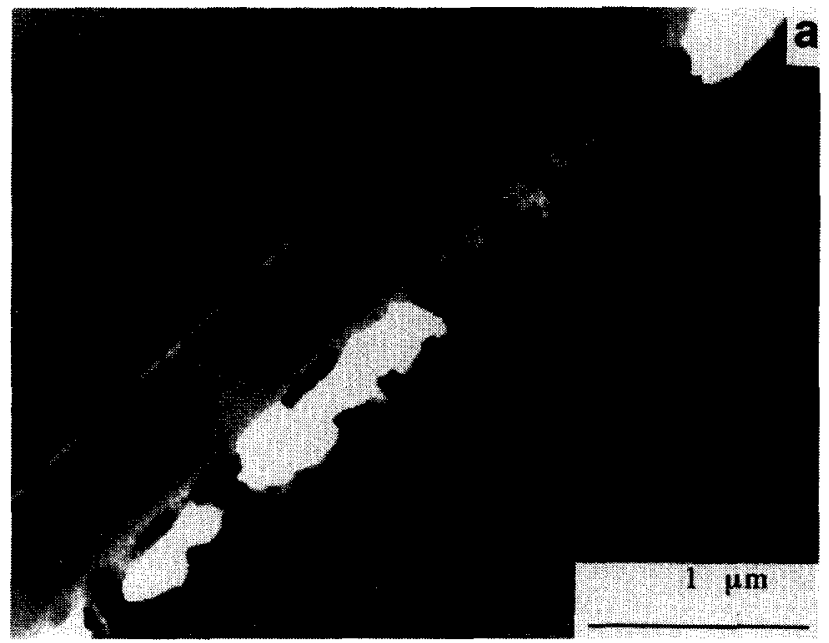

voids). Subsequently, all TEM micrographs and analyses were conducted in the loaded condition only.

Figures $l c$ and $l d$ show the TEM micrographs of $75 \%$ PS $/ 25 \%$ PPO and $50 \%$ PS $/ 50 \%$ PPO blends, respectively. Figure $1 \mathrm{c}$ shows the well developed craze microstructure of the $75 \%$ PS/25\% PPO blend. Figure $1 d$ shows a craze (located in the centre) surrounded by shear DZs (outer zones of craze with no voids). In some cases, $25 \%$ PPO inclusion resulted in the formation of shear $\mathrm{DZs}^{16}$. According to Donald and $\mathrm{Kramer}^{14}$, the shear DZ is not fibrillated as in a craze, but rather consists of polymers drawn to a uniform extension ratio, $\lambda_{\mathrm{DZ}}$. Donald and $\mathrm{Kramer}^{15}$ suggested that the shear DZ is thickened by drawing more polymers in from the edge of the zone, whereas crazes grow by fibrillation and formation of voids. The DZs (which are observed in thin films under the plane stress condition) grow along a direction normal to the tensile axis, whereas shear bands (which are observed in bulk samples under the plane strain condition) grow at about 45 to $58^{\circ}$ to the principal tensile axis. The DZs are also different from crazes in that they do not have any voiding and fibrillation. In $\mathrm{DZs}$, there are no sharp boundaries between the DZs and the undeformed film (Figure 1d). However, the crazes have sharp boundaries relative to the undeformed film (Figure 1c). The shear DZ is highly oriented and its fibril extension ratio can be measured using the same equation for craze fibril extension ratio as that developed by Lauterwasser and Kramer ${ }^{11}$.

According to Donald and Kramer ${ }^{6}$ polymers that have shorter entanglement molecular length $\left(l_{\mathrm{e}}\right)$ should have shear DZs, in contrast to polymers with longer $l_{e}$, which should show crazes. Polymers of intermediate $l_{e}$ show both crazes and shear DZs in their microstructural deformation. Later, this entanglement model will be applied to the analysis of the $\lambda$ results. Compared to the $75 \%$ PS $/ 25 \%$ PPO blend (Figure $1 c$ ), Figure $1 d$ has more shear DZs. As the PPO content increases, the frequency of these DZs increases as well. Figure le shows the shear $\mathrm{DZ}$ of a $25 \% \mathrm{PS} / 75 \% \mathrm{PPO}$ blend. In this case, a small trace of craze formation in the centre of the shear $\mathrm{DZ}$ (horizontal white line) is observed, and the majority of the deformation is shear-DZ-controlled. Figure If shows a shear DZ of $100 \%$ PPO, and the deformation is totally controlled by the shear DZ. Thus, from the TEM observation of PS/PPO blends, deformation mode

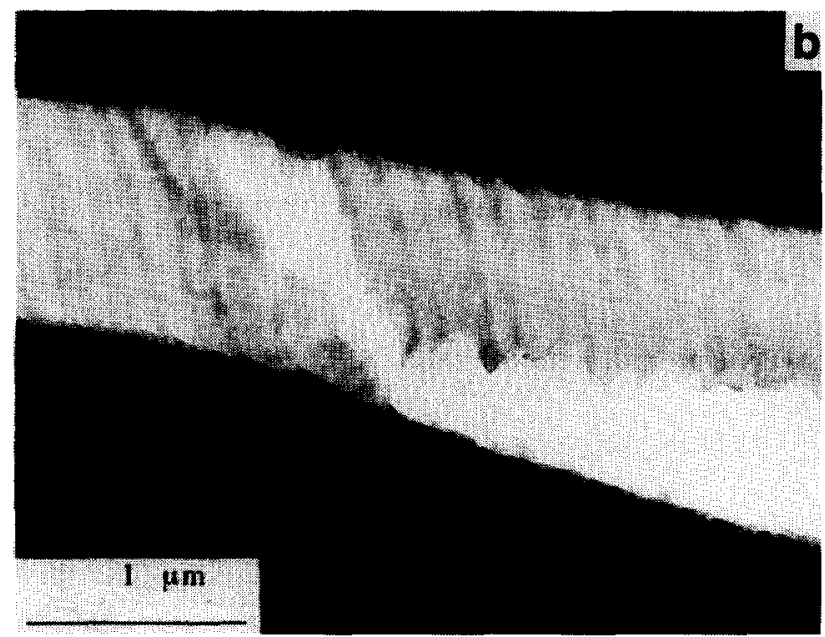

Figure 1 TEM micrographs of: (a) 100\% PS (unloaded condition); (b) 100\% PS (loaded condition); 

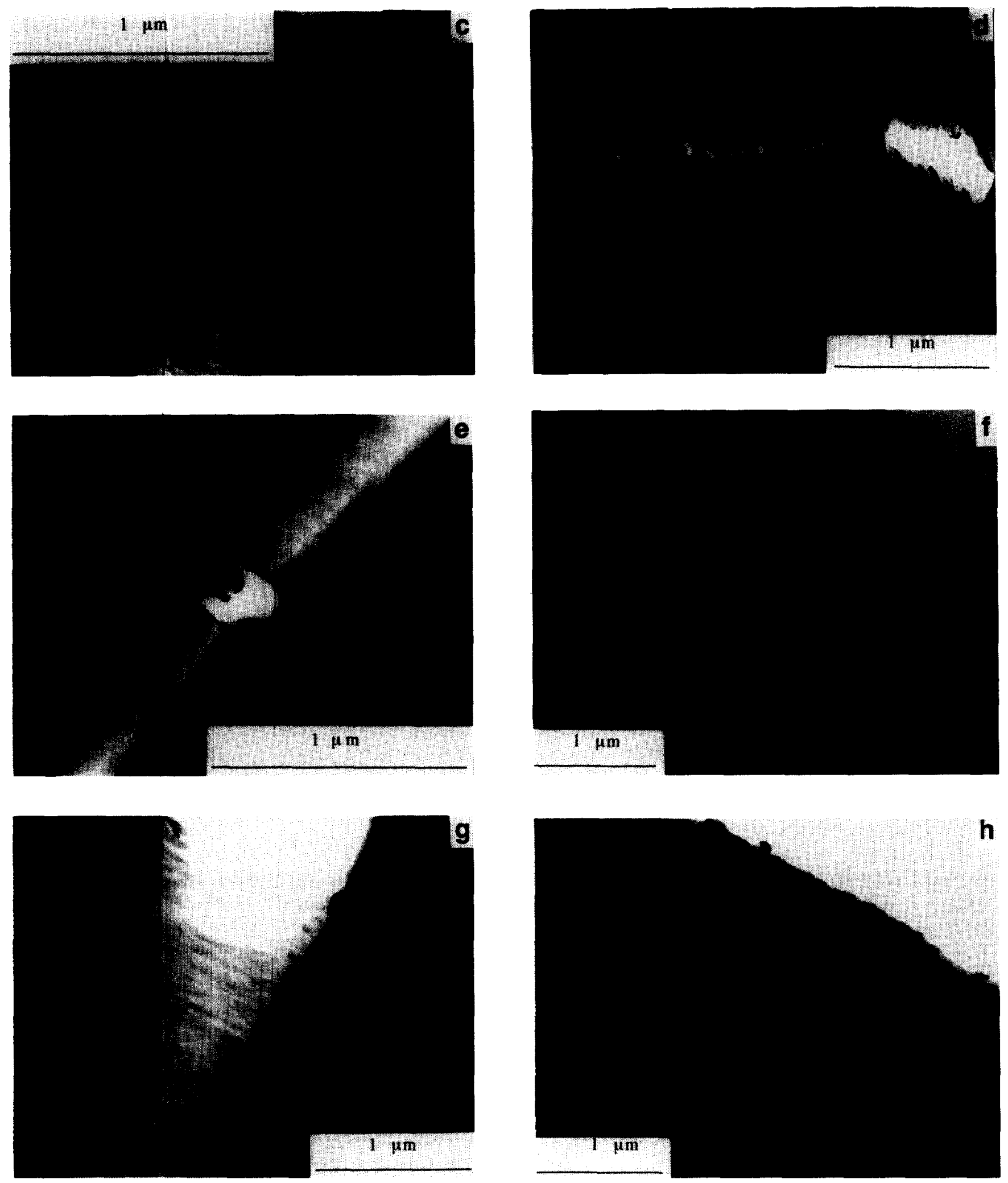

Figure 1 continued (c) $75 \%$ PS $/ 25 \%$ PPO blend; (d) 50\% PS $/ 50 \%$ PPO blend (notice craze surrounded by shear DZs); (e) $25 \%$ PS/75\% PPO blend (notice small trace of craze formation in the centre of shear DZ); (f) 100\% PPO; (g) annealed $25 \%$ PS $/ 75 \%$ PPO blend (notice craze surrounded by shear DZs); (h) annealed $100 \%$ PPO

transition from crazing to shear $\mathrm{DZ}$ was observed around $25 \%$ PPO inclusion.

\section{PS/PVME blends}

Figure 2 shows TEM micrographs of the PS/PVME blends. In the case of the $95 \%$ PS $/ 5 \%$ PVME blend (Figure $2 a$ ) and $85 \%$ PS/15\% PVME blend (Figure $2 b$ ), the deformation mode is craze-controlled. However, the $80 \%$ PS/20\% PVME blend (Figure $2 c$ ) shows both crazes (left of the crack) and shear DZs (right of the crack). In the case of a $65 \%$ PS $/ 35 \%$ PVME blend (Figure $2 d$ ), the deformation mode is almost entirely shear-DZ-controlled with a trace of craze formation. Thus, the deformation mode change from crazing to shear $\mathrm{DZ}$ occurs around $20 \%$ PVME inclusion in PS/PVME blends. 

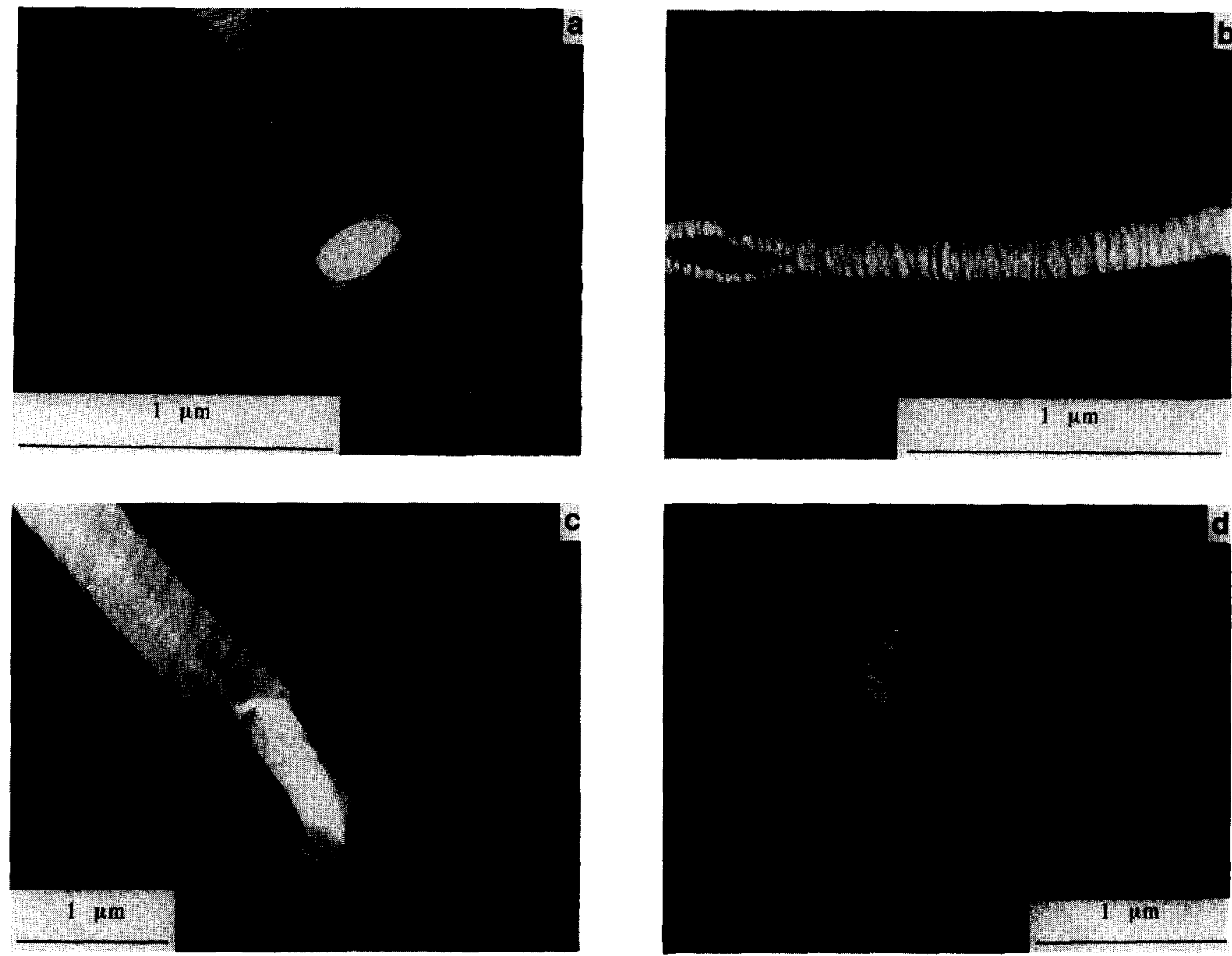

Figure 2 TEM micrographs of: (a) $95 \%$ PS $/ 5 \%$ PVME blend; (b) $85 \%$ PS $/ 15 \%$ PVME blend; (c) $80 \%$ PS $/ 20 \%$ PVME blend (notice craze left to crack and shear DZ right to crack); (d) $65 \% \mathrm{PS} / 35 \% \mathrm{PVME}$ blend (notice majority of deformation is shear $\mathrm{DZ}$ with a trace of craze formation)

\section{$P S / P \alpha M S$ and $P P O / P \alpha M S$ blends}

Figure 3 shows TEM micrographs of $75 \%$ PS $/ 25 \%$ $\mathrm{P} \propto \mathrm{MS}$ and $50 \% \mathrm{PS} / 50 \% \mathrm{P} \alpha \mathrm{MS}$ blends. Generally, the craze microstructures of PS/P $\alpha$ MS blends have coarser fibril and void structures than PS/PPO blends of the same compositions (Figure $3 a$ ). In other words, in these $\mathrm{PS} / \mathrm{P} \alpha \mathrm{MS}$ blends, the fibril volume fraction is much lower than that in the corresponding PS/PPO blends. For example, Figure $3 b$ shows the craze microstructure of a $50 \% \mathrm{PS} / 50 \% \mathrm{P} \alpha \mathrm{MS}$ blend. At this composition, the craze fibrils become too weak to form stable crazes, thereby resulting in a very coarse and easily disrupted craze microstructure. Thus, in PS/P $\alpha$ MS blends, the deformation mode is totally controlled by crazing. Figure 4 shows TEM micrographs of $75 \%$ PPO $/ 25 \%$ $\mathrm{P} \alpha \mathrm{MS}$ and $50 \% \mathrm{PPO} / 50 \% \mathrm{P} \alpha \mathrm{MS}$ blends. Figure $4 a$ shows the shear $\mathrm{DZ}$ of a $75 \% \mathrm{PPO} / 25 \% \mathrm{P} \alpha \mathrm{MS}$ blend and Figure $4 b$ shows the craze of a $50 \% \mathrm{PPO} / 50 \% \mathrm{P} \alpha \mathrm{MS}$ blend and crack propagation through the craze. In some cases, $25 \% \mathrm{P} \alpha \mathrm{MS}$ inclusion caused the blend to have a craze at this composition ${ }^{16}$. Thus, in PPO/P $\alpha$ MS blends, the deformation mode change from shear DZ to crazing occurs around $25 \% \mathrm{P} \alpha \mathrm{MS}$ inclusion.

The $\lambda$ results for all blend samples

Using a technique developed by Lauterwasser and Kramer $^{11}, \lambda$ of the PS/PPO blends were measured.
Table 3 lists the $\lambda$ results of this investigation with those of Donald and $\mathrm{Kramer}^{14}$. As shown, our result for $\lambda_{\text {craze }}$ for $100 \%$ PS sample is 4.3, in comparison with Donald and Kramer's result of 4.0. Considering the sensitivity of the microdensitometer, these values are in excellent agreement. For the $75 \% \mathrm{PS} / 25 \% \mathrm{PPO}$ blend, there is a difference between our result of 4.0 and Donald and Kramer's of 3.2. It is somewhat difficult to determine the reason for the discrepancy. For the 50\% PS $/ 50 \%$ PPO blends, the discrepancy is reduced. The $\lambda_{\mathrm{Dz}}$ values of the $75 \%$ PS $/ 25 \%$ PPO and $50 \%$ PS $/ 50 \%$ PPO blends showed a similarity for both groups. In addition, all the $\lambda_{\mathrm{DZ}}$ values were of the order of 2 regardless of the blend composition in PS/PPO blends. For the $25 \%$ PS/75\% PPO blend and $100 \%$ PPO samples, no crazes are observed under normal conditions. However, the above authors grew crazes artificially by annealing the samples. Later, using similar conditions to Donald and $\mathrm{Kramer}^{6}$, the $25 \% \mathrm{PS} / 75 \%$ PPO blend and $100 \%$ PPO sample were annealed. Figures $l g$ and $l h$ show the TEM micrographs of annealed $25 \%$ PS $/ 75 \%$ PPO blend and $100 \%$ PPO, respectively. Figure $1 g$ shows both a craze (extended fibrils and voids) and a shear DZ (surrounding the craze with no void formation) simultaneously, similar to Figure 1d. The annealed 100\% PPO (Figure 1h), however, still shows only shear DZs in this investigation. The $\lambda$ value of annealed $25 \%$ PS $/ 75 \%$ PPO blend was 

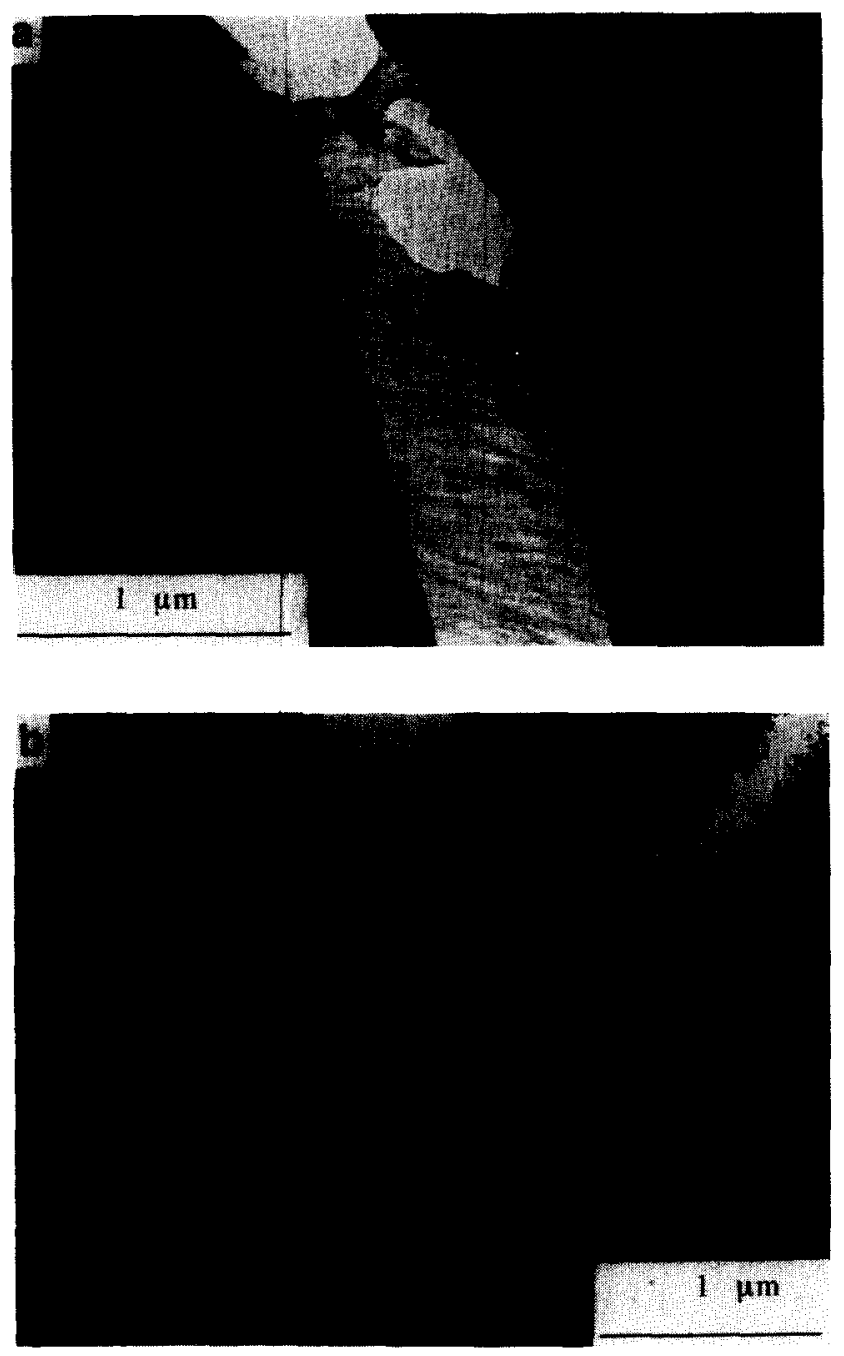

Figure 3 TEM micrographs of: (a) $75 \%$ PS/25\% P $\alpha$ MS blend; (b) $50 \% \mathrm{PS} / 50 \% \mathrm{P} \alpha \mathrm{MS}$ blend

3.4 for the craze and 2.4 for the DZ. For the $100 \%$ PPO, $\lambda_{\mathrm{DZ}}$ was around 2.1 , approximately the same as what was observed for unannealed $100 \%$ PPO.

Figure 5 shows the plot of the average $\lambda$ values vs. composition of other blends along with PS/PPO blends. Evidently the trend is as follows: for PS/PPO and PS/PVME blends, $\lambda$ decreased as PPO and PVME content increased; for PS/P $\alpha M S$ and $P P O / P \alpha M S$ blends, $\lambda$ increased as the $\mathrm{P} \alpha \mathrm{MS}$ content increased. These overall $\lambda$ results were analysed using the chain entanglement model in the next section.

Application of chain entanglement model to the analyses of $\lambda$ results

In the early days, the chain entanglement concept was used primarily to explain the rheological behaviour of polymer melts and concentrated polymer solutions. Aharoni ${ }^{17}$ expanded on these ideas and estimated the number of chain atoms between entanglements for a large number of polymers. However, the effect of entanglement is also very significant in the glassy state. For example, Gent and Thomas ${ }^{18}$ showed the existence of a relationship between tensile strength, molecular weight and entanglement molecular weight in the glassy state of amorphous polymers. Robertson ${ }^{19}$ showed that, for polymers with a molecular weight less than $M_{\mathrm{e}}$, the fracture toughness decreased catastrophically, resulting in a low tensile strength due to fibril instability in the craze.

\section{PS/PPO blends}

Donald and Kramer recognized the relationship between chain entanglement and craze fibril stability ${ }^{15,20}$. Using a series of polymers of different entanglement molecular weights, these authors showed that the
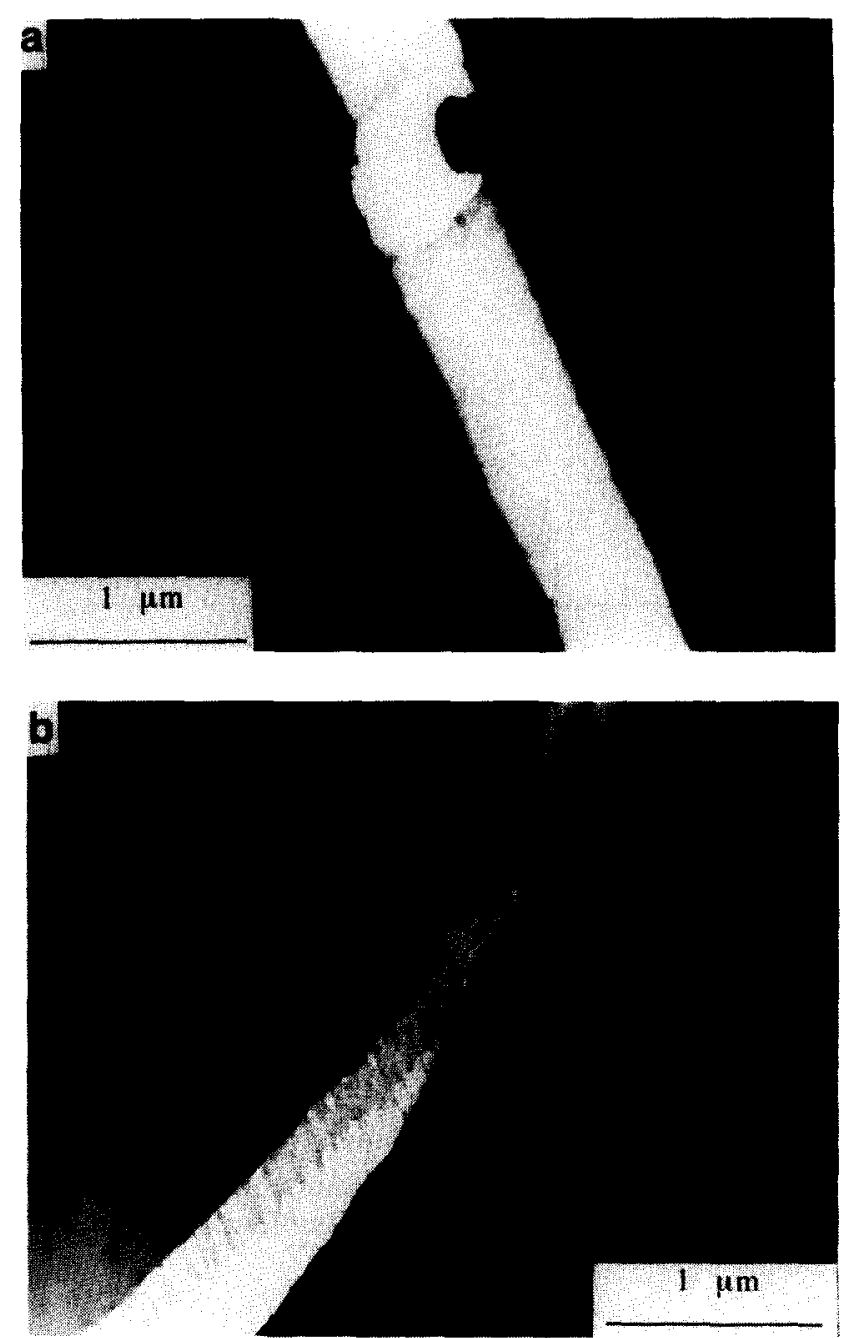

Figure 4 TEM micrographs of: (a) $75 \% \mathrm{PPO} / 25 \% \mathrm{P} \alpha \mathrm{MS}$ blend (shear DZ); (b) $50 \% \mathrm{PPO} / 50 \% \mathrm{P} \alpha \mathrm{MS}$ blend (craze formation and crack propagation through craze)

Table 3 Comparison of $\lambda$ values of PS/PPO blends from Donald and Kramer $^{14}$ with those from this investigation

\begin{tabular}{lll}
\hline & $\lambda_{\text {craze }}$ & $\lambda_{\mathrm{DZ}}$ \\
\hline Donald and Kramer's results & & \\
$100 \%$ PS & 4.0 & - \\
$75 \%$ PS $/ 25 \%$ PPO & 3.2 & 2.1 \\
$50 \%$ PS $/ 50 \%$ PPO & $2.8^{a}$ & 2.0 \\
$25 \%$ PS/75\% PPO & $3.4^{a}$ & 1.6 \\
$100 \%$ PPO & $2.6^{a}$ & 1.6 \\
This investigation & & \\
$100 \%$ PS & 4.3 & - \\
$75 \%$ PS/25\% PPO & 4.0 & 2.1 \\
$50 \%$ PS/50\% PPO & 3.1 & 2.1 \\
$25 \%$ PS/75\% PPO & $3.4^{a}$ & 2.1 \\
$100 \%$ PPO & - & \\
\hline
\end{tabular}

${ }^{a}$ Annealed sample 


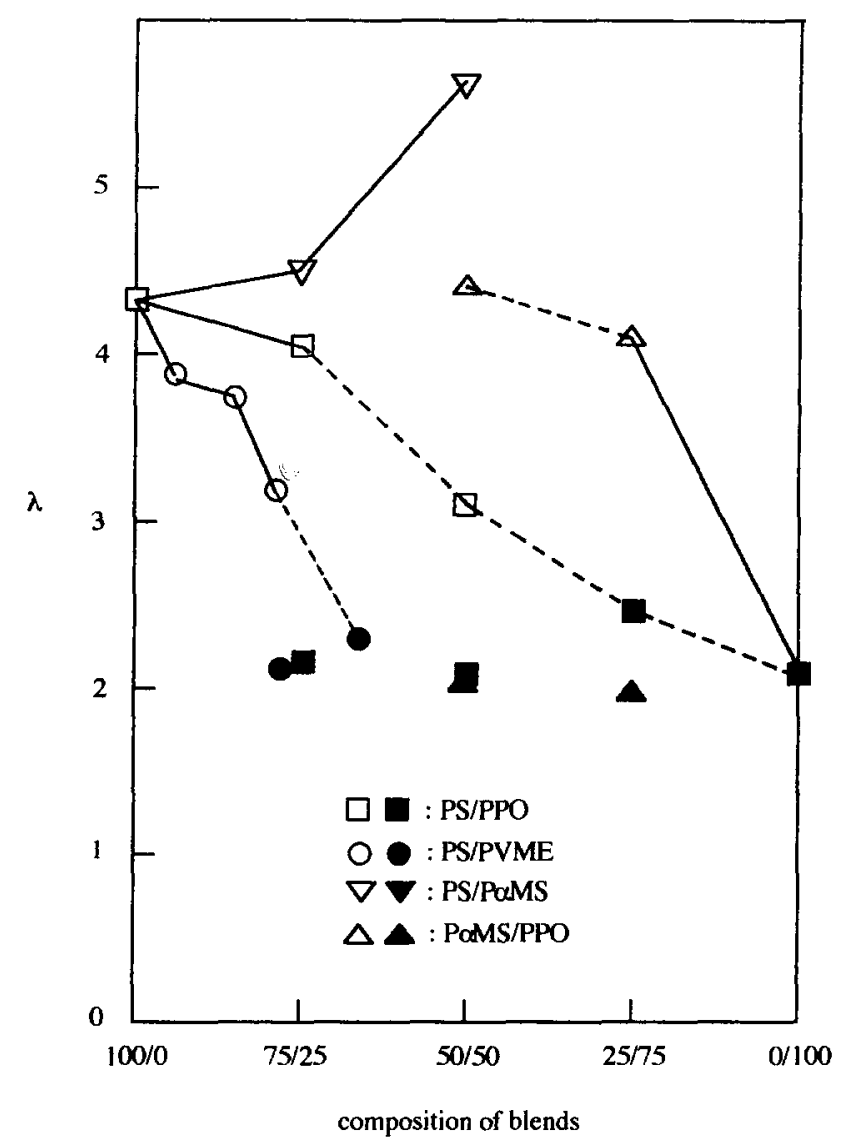

Figure 5 Fibril extension ratios of all blend samples (open symbols indicate craze; filled symbols indicate DZ)

difference in craze microstructure represents the difference in the entanglement network in their polymers. They used PS/PPO blends to test their entanglement model on the deformation behaviour. Their model is based on the assumption that the theoretical maximum fibril extension ratio can be represented by $\lambda_{\max }=l_{\mathrm{e}} / d$. Here, $l_{\mathrm{e}}$ is the chain contour length between entanglements and $d$ is the entanglement mesh size. This latter quantity can be calculated from the root-mean-square end-to-end distance for a polymer chain of molecular weight $M_{\mathrm{e}}$ using:

$$
d=\kappa M_{\mathrm{e}}^{1 / 2}
$$

where $\kappa$ is a polymer-dependent constant, which may be determined directly from neutron scattering measurements of the radius of gyration of molecular coils in the glassy state or from light scattering measurements of molecular coil size in dilute solution in a $\theta$ solvent. The above authors made several assumptions for this model.

First, they assumed a value of $M_{\mathrm{e}}$ for PS of $19100 \mathrm{~g} \mathrm{~mol}^{-1}$ from melt elasticity measurements. Then, the quantity $l_{\mathrm{e}}$ can be calculated from:

$$
l_{\mathrm{e}}=l_{\mathrm{o}}(v) M_{\mathrm{e}}(v) / M_{0}
$$

where $1_{0}$, the length of a fully extended chain unit of molecular weight $M_{0}$, was determined from the crystallographic data and $v$ is the volume fraction of PPO. A value of $400 \AA$ was reported. They also used an equation developed by Prest and Porter ${ }^{21}$ for calculating the entanglement molecular weight of PS/PPO blends. The entanglement molecular weight $M_{\mathrm{e}}(w)$ can be calculated using the following equation:

$$
M_{\mathrm{e}}(w)=M_{\mathrm{c}}(\mathrm{PS}) /(1+3.2 w)
$$

where $w$ represents the weight fraction of PPO. Donald and Kramer ${ }^{15,20}$ extrapolated the value of $M_{\mathrm{e}}(w=1.00)$ for PPO from equation (3), and obtained a value of $4300 \mathrm{~g} \mathrm{~mol}^{-1}$, and a corresponding value for $l_{\mathrm{e}}$ of $165 \AA$.

Secondly, they used a simple rule of mixtures to describe the variation of $l_{0} / M_{0}$ for the blends and obtained the following equation:

$$
l_{\mathrm{e}}=M_{\mathrm{e}}(\mathrm{PS})(0.0209+0.0175 w) /(1+3.2 w)
$$

From equation (1), they were able to calculate the $d$ values for PS and PPO, and obtained a $d$ value of $96 \AA$ for PS and $55 \AA$ for PPO.

Thirdly, again using a simple rule of mixtures to describe the values of $d$ for the blends, they derived the following equation:

$$
d(w)=96-41 w(\AA)
$$

Using equation (5), they calculated the $d$ values of the PS/PPO blends. Finally, by combining equations (4) and (5), Donald and Kramer obtained the theoretical $\lambda_{\max }$ values for PS/PPO blends.

However, the assumptions made to derive the above equations could be criticized because the effect of intermolecular interactions on the chain entanglements was ignored. The above model is based on topological entanglements (a special type of intermolecular interactions) without considering the effect of interaction points caused by thermodynamically favourable intermolecular interactions in compatible blends. For example, the $d$ values of blends were calculated from equation (5), which is based on a simple rule of mixtures. In real PS/PPO blends, the existence of this linear relationship of $d$ values is difficult owing to the favourable interactions between the PS and PPO chains. In a hypothetical $\theta$ solvent where there are neither favourable nor unfavourable interactions $(\chi=0)$, this linear relationship might be acceptable. However, real PS chains are a good solvent for PPO chains and vice versa. Thus the real end-to-end mesh size in PS/PPO blends should be larger in comparison to its value in the $\theta$ solvent. Maconnachie et $a .^{22}$ studied the small-angle neutron scattering behaviour of PS/PPO blends. They found a negative interaction parameter $\chi$ for all PS/PPO blends, indicating a thermodynamically stable mixture. Thus, in PS/PPO blends, the radius of gyration should be larger than in the $\theta$ condition. This indicates that the linear assumption of $d$ in Donald and Kramer's treatment is not an accurate one. Thus, $\lambda$ of compatible blends cannot be fully explained by the chain entanglement model alone. There should be a consideration of intermolecular interactions, which affect the topological entanglements.

Recently, $\mathrm{Wu}^{23}$ studied the entanglement, friction and free-volume behaviour in a series of compatible blends including PS/PPO blends. $\mathrm{Wu}$ found that the intermolecular interaction, which is responsible for the compatibility of blends, tends to reduce the entanglement but increase the friction between dissimilar chains. Wu explains that the specific intermolecular interaction tends to align the chain segments to maximize interactions, thus stiffening the segments locally, reducing their convolution and producing a reduction in entanglements between dissimilar chains. This behaviour means that, in equation (2), $l_{\mathrm{e}}$ becomes larger since $M_{\mathrm{e}}$ increases due to reduced entanglements. However, $d$ values are also supposed to be larger since coil dimensions expand owing to the effect of a good solvent. Thus, it is 
somewhat difficult to determine the real effect of specific intermolecular interactions on $\lambda$ since both $d$ and $l_{e}$ are supposed to be larger simultaneously owing to the intermolecular interactions. However, it is certain that the $\lambda$ of PS/PPO blends cannot be explained simply by using the entanglement model alone. Specific intermolecular interactions, which lead to the change of $d$ and $l_{e}$ values, should be another important parameter affecting $\lambda$ values. Nevertheless, Donald and Kramer's simple model gives qualitatively agreeable explanations on the fibril extension ratio behaviour in PS/PPO blends. However, there should be caution in accepting the entanglement model as a sole contributor to $\lambda$ behaviour and further microstructural deformation behaviour of compatible blends.

\section{$P S / P V M E$ blends}

For the PS/PVME blends, the fibril extension ratio decreases as the PVME content increases. This behaviour can be qualitatively explained by considering the entanglement molecular weight of PVME homopolymer. The critical molecular weight $M_{\mathrm{c}}$ of PVME is known to be about $17000 \mathrm{~g} \mathrm{~mol}^{-1}$. Considering the fact that $M_{\mathrm{c}}$ is generally about $2 M_{\mathrm{e}}$, the $M_{\mathrm{e}}$ of PVME should be about 8000 to $9000 \mathrm{~g} \mathrm{~mol}^{-1}$. By blending PS with PVME, there should be an increase of entanglement density and a corresponding decrease of entanglement molecular weight in PS/PVME blends. Considering the entanglement molecular weight of PPO $\left(M_{\mathrm{e}}=4000 \mathrm{~g} \mathrm{~mol}^{-1}\right)$, there should be a lower $\lambda$ in PS/PPO blends compared to PS/PVME blends from the viewpoint of the entanglement model. By comparing $\lambda$ of PS/PPO and PS/PVME blends of similar composition, the PS/PVME blend is seen to have a lower $\lambda$. Clearly, this behaviour cannot be explained by an entanglement model alone. Kambour et al. $^{24}$ investigated the interaction forces between PS/PPO blends. Kwei et al..$^{25}$ and Su and Patterson ${ }^{26}$ studied the interaction forces between PS/PVME blends. Both groups characterized the interaction force in terms of the $\chi$ parameter. For PS/PPO blends, $\chi$ was around -1 , whereas, for PS/PVME blends, $\chi$ was between 0 and -0.4 . This indicates that the interaction force of PS/PVME blends is weaker than that of PS/PPO blends. Thus, the $\lambda$ results of PS/PVME blends cannot be explained successfully in terms of either entanglement or intermolecular interaction model.

Faivre et al. ${ }^{27}$ studied the orientation and relaxation behaviour of PS/PPO blends and PS/PVME blends. They found that PS chains in PS/PPO and PS/PVME blends acquired a higher degree of orientation in both blends compared with the pure PS chains. On the other hand, PPO chains were highly oriented in PS/PPO blends, while PVME chains remained almost unoriented in PS/PVME blends. These workers explained that the specific intermolecular interactions modified the molecular environment of polymer chains. Thus, the mobility of the two polymer chains is hindered and the friction coefficient of each species is increased. They concluded that PVME chains, which had a very fast relaxation time, remained unoriented, while PPO chains kept the orientation that they acquired during stretching since their relaxation times are very long. Thus the reason why PS/PVME blends have lower $\lambda$ than those of PS/PPO blends of the same composition might be the relaxation-time difference between PPO and PVME chains. In other words, after stretching the PS/PVME blends, the PVME chains relax much faster than the PPO chains and quickly return to the relaxed, coiled chain configuration. This intermolecular-interaction-induced relaxation of PVME chains might account for the observed lower $\lambda$ values of PS/PVME blends in contrast to those of PS/PPO blends of the same composition.

\section{$P S / P \propto M S$ blends}

For the PS/P $\alpha \mathrm{MS}$ blends, $\lambda$ increased as the P $\alpha \mathrm{MS}$ content increased. From the viewpoint of the chain entanglement model, the molecular weight of $\mathrm{P} \alpha \mathrm{MS}$ used in this study is $50000 \mathrm{~g} \mathrm{~mol}^{-1}$, which is approximately the same as its $M_{\mathrm{c}}$. Thus, by mixing $\mathrm{P} \alpha \mathrm{MS}$ with PS, there is a dilution of entanglements since the P $\alpha$ MS does not contribute to the formation of a stable entanglement network. This dilution of entanglement network results in a higher $\lambda$. From the thermodynamic intermolecular interactions of PS/P $\alpha \mathrm{MS}$ blends, it is well known that the interactions between the PS and $P \alpha M S$ chains are less than those between the PS and PPO chains owing to the existence of the methyl group in $\mathrm{P} \alpha \mathrm{MS}^{10}$. A separate experiment conducted by Choe and Aklonis ${ }^{28}$ confirms the above observation. Measurement of $\lambda$ indicates an increase in the fibril extension ratio as the $\mathrm{P} \alpha \mathrm{MS}$ content increases. Thus, from an interaction point of view, the fibrillation procedure of PS/P $\alpha \mathrm{MS}$ blends should be easier owing to the lower interactions compared to PS/PPO blends, which may result in less hindrance to the fibrillation process than the PS/PPO blends. Thus, $\lambda$ results of PS/P $\alpha$ MS blends can be explained in terms of combined entanglement and intermolecular interaction model.

\section{PPO/P $\alpha M S$ blends}

For the PPO/P $\propto \mathrm{MS}$ blends, the above argument can be applied. Compared to the PPO/PS blends, the intermolecular interactions of PPO/P $\alpha \mathrm{MS}$ blends should be smaller owing to the presence of methyl groups in $\mathrm{P} \alpha \mathrm{MS}^{10}$. The lower intermolecular interactions may lead to smaller friction between PPO and P $\alpha$ MS chains. This decreased friction coefficient should then lead to less hindrance to the fibrillation process than the PPO/PS blends, which, in turn, results in higher fibril extension ratios in the PPO/P $\alpha$ MS blends compared to those in the PPO/PS blends. From the entanglement point of view, the $\lambda$ behaviour of PPO/P $\alpha$ MS blends can be explained by PPO entanglement dilution. By adding $\mathrm{P} \alpha \mathrm{MS}$, the existing PPO entanglements are diluted since $P \propto M S$ cannot contribute to the formation of stable entanglements owing to its low molecular weight. This entanglement dilution causes the observed higher $\lambda$ in the PPO/P $\propto \mathrm{MS}$ blends. Thus, the $\lambda$ results of $\mathrm{PPO} / \mathrm{P} \propto \mathrm{MS}$ blends can be explained in terms of combined entanglement and intermolecular interaction model.

During the measurement of $\lambda$ of crazes and shear DZs, one interesting observation is that $\lambda_{\mathrm{DZ}}$ values are always of the order of 2 regardless of blend types or compositions. This phenomenon is probably due to the fact that craze formation requires complete fibrillation and orientation of chains with void formation, whereas shear DZ formation does not involve any void formation or complete fibrillation and orientation. This might account for the lower $\lambda_{\mathrm{DZ}}$ values than $\lambda_{\text {craze }}$ values. Also, by comparing these $\lambda$ values of shear DZ with the shear strain obtained by Chau and $\mathrm{Li}^{29}$ from the shear bands 
in bulk PS, an interesting result was obtained. The above authors obtained a shear strain of unity in the bulk shear band, indicating that $\lambda$ of the shear band has a value of 2 since $\lambda=e+1$. Our $\lambda$ values of the shear $\mathrm{DZ}$ were around 2 , which is a similar value to that obtained from the shear bands in the bulk sample.

From the above analyses, it is concluded that the fibril extension ratio behaviour of compatible blends can be generally explained by the combined effect of entanglement and intermolecular interaction models. In the case of PS/PVME blends, the $\lambda$ results cannot be successfully explained by the above models, rather by the intermolecular-interaction-induced relaxation of PVME chains.

\section{SUMMARY}

The individual craze and shear $\mathrm{DZ}$ microstructures were observed by TEM and quantitatively analysed by microdensitometry. From the TEM micrographs, 25\% PPO inclusion resulted in a deformation mode change from crazing to shear DZ in PS/PPO blends. For PS/PVME blends, this transition occurred around $25 \%$ PVME inclusion. For PS/P $\alpha$ MS blends, deformation mode was controlled by crazing only. For PPO/P $\alpha \mathrm{MS}$ blends, the deformation mode transition from shear $\mathrm{DZ}$ to crazing occurred around $25 \% \mathrm{P} \alpha \mathrm{MS}$ inclusion. One parameter that characterizes craze or shear DZ is the fibril extension ratio, $\lambda_{\text {craze }}$ or $\lambda_{\mathrm{DZ}}$. The $\lambda_{\text {craze }}$ values of all blends showed the following behaviour: in the PS/PPO and PS/PVME blends, $\lambda_{\text {craze }}$ decreased as the PPO and PVME content increased; in the PS/P $\alpha \mathrm{MS}$ and $\mathrm{PPO} / \mathrm{P} \alpha \mathrm{MS}$ blends, $\lambda_{\text {craze }}$ increased as the $\mathrm{P} \alpha \mathrm{MS}$ content increased. The $\lambda_{\mathrm{DZ}}$ values of all blends were always of the order of 2 regardless of blend types and compositions.

The fibril extension ratio behaviour was first analysed in terms of the entanglement model developed by Donald and Kramer. However, this model cannot fully explain $\lambda_{\text {craze }}$ or $\lambda_{\mathrm{Dz}}$ behaviour in compatible blends owing to the inherent limitations in the assumption stages. Rather, the microstructural deformation behaviours of PS/PPO, $\mathrm{PS} / \mathrm{P} \alpha \mathrm{MS}$ and $\mathrm{PPO} / \mathrm{P} \alpha \mathrm{MS}$ blends can be generally explained using the combined model of entanglement density and intermolecular interaction model. For PS/PVME blends, the deformation behaviour can be explained in terms of intermolecular-interaction-induced relaxation of PVME chains.

\section{ACKNOWLEDGEMENTS}

Financial support of this work by the National Science Foundation is gratefully acknowledged. One of the authors (B. C. Chun) would like to thank the Macromolecular Research Center for providing a fellowship during this work.

\section{REFERENCES}

Cizek, E. P., US Patent 3383435, 1968

Yee, A. F. Polym. Eng. Sci. 1977, 17, 213

Wellinghoff, S. T. and Baer, E. J. Appl. Polym. Sci. 1978, 22, 2025

Argon, A. S. Phil. Mag. 1973, 28, 1839

Yeh, G. S. Y. J. Macromol. Sci.-Phys. (B) 1973, 7, 729

Donald, A. M. and Kramer, E. J. Polymer 1982, 23, 461

Stoelting, J., Karasz, F. E. and Macknight, W. J. Polym. Eng. Sci. 1970, 10, 133

8 Lu, F. J., Burchell, D. J., Li, X. and Hsu, S. L. Polym. Eng. Sci $1983,23,861$

9 Dunn, D. S. and Krause, S. J. Polym. Sci., Polym. Lett. Edn. $1974,12,591$

10 Mikes, F., Morawetz, H. and Dennis, K. S. Macromolecules 1980 , 13, 969

11 Lauterwasser, B. D. and Kramer, E. J. Phil. Mag. 1979, 39, 469

12 Brown, H. R. and Kramer, E. J. J. Macromol. Sci.-Phys. (B) $1981,19,487$

13 Kambour, R. P. J. Polym. Sci. (D) 1973, 7, 1

14 Donald, A. M. and Kramer, E. J. J. Mater. Sci. 1982, 17, 1871

15 Donald, A. M. and Kramer, E. J. J. Mater. Sci. 1981, 16, 2967

16 Chun, B. and Gibala, R. unpublished data

17 Aharoni, S. M. Macromolecules 1983, 16, 1722

18 Gent, A. N. and Thomas, A. G. J. Polym. Sci., Polym. Phys. Edn. 1972, 10, 571

19 Robertson, R. E. 'Toughness and Brittleness of Plastics' in Advances in Chemistry Series, American Chemical Society, Washington, DC, 1976 , p. 89

20 Donald, A. M. and Kramer, E. J. J. Mater. Sci. 1981, 16, 2677

21 Prest, Jr, W. M. and Porter, R. S. J. Polym. Sci., Polym. Phys. Edn. 1972, 10, 1639

22 Maconnachie, A. K., Kambour, R. P., White, D. M. and Walsh, D. J. Macromolecules 1984, 17, 2645

Wu, S. J. Polym. Sci., Polym. Phys. Edn. 1987, 25, 2511

24 Kambour, R. P., Bendler, J. T. and Bopp, R. C. Macromolecules 1983, 16, 753

25 Kwei, T. K., Nishi, T. and Roberts, R. F. Macromolecules 1974 7, 667

Su, C. S. and Patterson, D. Macromolecules 1977, 10, 708 Faivre, J. P., Jasse, B. and Monnerie, R. F. Polymer 1985, 26, 879 Choe, S. and Aklonis, J. J. Polym. Eng. Sci. 1987, 27, 1284 Chau, C. C. and Li, J. C. M. J. Mater. Sci. 1979, 14, 2172 